\title{
Some Further Generalizations of Hölder's Inequality and Related Results on Fractal Space
}

\author{
Guang-Sheng Chen, ${ }^{1}$ H. M. Srivastava, ${ }^{2}$ Pin Wang, ${ }^{3}$ and Wei Wei ${ }^{4}$ \\ ${ }^{1}$ Department of Construction and Information Engineering, Guangxi Modern Vocational Technology College, Hechi, \\ Guangxi 547000, China \\ ${ }^{2}$ Department of Mathematics and Statistics, University of Victoria, Victoria, BC, Canada V8W 3R4 \\ ${ }^{3}$ Department of Mathematics and Computer Science, Guangxi College of Education, Nanning, Guangxi 530023, China \\ ${ }^{4}$ School of Computer Science and Engineering, Xian University of Technology, Xian, Shaanxi 710048, China
}

Correspondence should be addressed to Pin Wang; 1040168586@qq.com

Received 16 May 2014; Accepted 21 June 2014; Published 6 July 2014

Academic Editor: Xiao-Jun Yang

Copyright (c) 2014 Guang-Sheng Chen et al. This is an open access article distributed under the Creative Commons Attribution License, which permits unrestricted use, distribution, and reproduction in any medium, provided the original work is properly cited.

We establish some new generalizations and refinements of the local fractional integral Hölder's inequality and some related results on fractal space. We also show that many existing inequalities related to the local fractional integral Hölder's inequality are special cases of the main inequalities which are presented here.

\section{Introduction}

Let $p_{j}(j=1,2, \ldots)$ be constrained by

$$
\sum_{j=1}^{m} \frac{1}{p_{j}}=1
$$

Suppose also that $f_{j}(x)>0$ and $f_{j}(j=1,2, \ldots, m)$ are continuous real-valued functions on $[a, b]$. Then each of the following assertions holds true.

(1) For $p_{j}>0(j=1,2, \ldots, m)$, we have the following inequality known as the Hölder inequality (see [1]):

$$
\int_{a}^{b} \prod_{j=1}^{m} f_{j}(x) d x \leqq \prod_{j=1}^{m}\left(\int_{a}^{b} f_{j}^{p_{j}}(x) d x\right)^{1 / p_{j}} .
$$

(2) For $0<p_{m}<1$ and $p_{j}<0(j=1,2, \ldots, m-1)$, we have the following reverse Hölder inequality (see [2]):

$$
\int_{a}^{b} \prod_{j=1}^{m} f_{j}(x) d x \geqq \prod_{j=1}^{m}\left(\int_{a}^{b} f_{j}^{p_{j}}(x) d x\right)^{1 / p_{j}} .
$$

In the special case when $m=2$ and $p_{1}=p_{2}$, inequality (2) reduces to the celebrated Cauchy inequality (see [3]). Both the
Cauchy inequality and the Hölder inequality play significant roles in many different branches of modern pure and applied mathematics. A great number of generalizations, refinements, variations, and applications of each of these inequalities have been studied in the literature (see [3-13] and the references cited therein). Recently, Yang [14] established the following local fractional integral Hölder's inequality on fractal space.

Let $f(x), g(x) \in C_{\alpha}(a, b), p>1$, and $1 / p+1 / q=1$. Then

$$
\begin{aligned}
& \frac{1}{\Gamma(1+\alpha)} \int_{a}^{b}|f(x) g(x)|(d x)^{\alpha} \\
& \leqq \\
& \quad\left(\frac{1}{\Gamma(1+\alpha)} \int_{a}^{b}|f(x)|^{p}(d x)^{\alpha}\right)^{1 / p} \\
& \quad \times\left(\frac{1}{\Gamma(1+\alpha)} \int_{a}^{b}|g(x)|^{q}(d x)^{\alpha}\right)^{1 / q} .
\end{aligned}
$$

More recently, Chen [15] gave a generalization of inequality (4) and its corresponding reverse form as follows.

Let $f_{j}(x) \in C_{\alpha}(a, b), p_{j} \in R(j=1,2, \ldots, m)$, and

$$
\sum_{j=1}^{m} \frac{1}{p_{j}}=1 .
$$


Then each of the following assertions holds true. (1) For $p_{j}>$ $1(j=1,2, \ldots, m)$, we have

$$
\begin{aligned}
& \frac{1}{\Gamma(1+\alpha)} \int_{a}^{b} \prod_{j=1}^{m}\left|f_{j}(x)\right|(d x)^{\alpha} \\
& \leqq \prod_{j=1}^{m}\left(\int_{a}^{b} \frac{1}{\Gamma(1+\alpha)}\left|f_{j}(x)\right|^{p_{j}}(d x)^{\alpha}\right)^{1 / p_{j}} .
\end{aligned}
$$

(2) For $0<p_{1}<1$ and $p_{j}<0(j=2, \ldots, m)$, we have

$$
\begin{aligned}
& \frac{1}{\Gamma(1+\alpha)} \int_{a}^{b} \prod_{j=1}^{m}\left|f_{j}(x)\right|(d x)^{\alpha} \\
& \geqq \prod_{j=1}^{m}\left(\int_{a}^{b} \frac{1}{\Gamma(1+\alpha)}\left|f_{j}(x)\right|^{p_{j}}(d x)^{\alpha}\right)^{1 / p_{j}} .
\end{aligned}
$$

The study of local fractional calculus has been an interesting topic (see [14-25]). In fact, local fractional calculus $[14,16,17]$ has turned out to be a very useful tool to deal with the continuously nondifferentiable functions and fractals. This formalism has had a great variety of applications in describing physical phenomena, for example, elasticity [17, 26, 27], continuum mechanics [26], quantum mechanics $[28,29]$, wave phenomena and heat-diffusion analysis [3034 , and other branches of pure and applied mathematics $[15,35-37]$ and nonlinear dynamics [38, 39]. For more details and other applications of local fractional calculus, the interested reader may refer to the recent works [14-42] (see also the monograph [43] dealing extensively with fractional differential equations).

The purpose of this paper is to give some new generalizations and refinements of inequalities (6) and (7). Some related inequalities are also considered. This paper is structured as follows. In Section 2, we introduce some basic facts about local fractional calculus. In Section 3, we establish some new generalizations and refinements of the local fractional integral Hölder inequality and their corresponding reverse forms. Finally, we give our concluding remarks and observations in Section 4.

\section{Preliminaries}

In this section, we recall some known results of local fractional calculus (see $[14,16,17])$. Throughout this section we will always assume that $F$ is a subset of the real line and is a fractal.

Lemma 1 (see [17]). Assume that $f:(F, d) \rightarrow\left(\Omega^{\prime}, d^{\prime}\right)$ is a biLipschitz mapping; then there are two positive constants $\rho, \tau$, and $F \subset R$,

$$
\rho^{s} H^{s}(F) \leqq H^{s}(f(F)) \leqq \tau^{s} H^{s}(F)
$$

such that

$$
\rho^{\alpha}\left|x_{1}-x_{2}\right|^{\alpha} \leqq\left|f\left(x_{1}\right)-f\left(x_{2}\right)\right| \leqq \tau^{\alpha}\left|x_{1}-x_{2}\right|^{\alpha}
$$

holds true for all $x_{1}, x_{2} \in F$.
Based on Lemma 1, it is easy to show that [14]

$$
\left|f\left(x_{1}\right)-f\left(x_{2}\right)\right| \leqq \tau^{\alpha}\left|x_{1}-x_{2}\right|^{\alpha},
$$

such that the following inequality holds true [14]:

$$
\left|f\left(x_{1}\right)-f\left(x_{2}\right)\right| \leqq \varepsilon^{\alpha},
$$

where $\alpha$ is fractal dimension of $F$.

Definition 2 (see $[14,17])$. Assume that $\varepsilon, \delta>0,\left|x-x_{0}\right|^{\alpha} \leqq \delta$, and $\varepsilon, \delta \in R$; if

$$
\left|f(x)-f\left(x_{0}\right)\right| \leqq \varepsilon^{\alpha},
$$

then $f(x)$ is called local fractional continuous at $x=x_{0}$, denoted by $\lim _{x \rightarrow x_{0}} f(x)=f\left(x_{0}\right)$. If $f(x)$ is local fractional continuous on the interval $(a, b)$, then we write (see, e.g., [14])

$$
f(x) \in C_{\alpha}(a, b),
$$

where $C_{\alpha}(a, b)$ denotes the space of local fractional continuous functions on $(a, b)$.

Definition 3 (see $[16,17]$ ). Suppose that $f(x)$ is a nondifferentiable function of exponent $\alpha(0<\alpha \leqq 1)$. If the following inequality holds true

$$
|f(x)-f(y)| \leqq C|x-y|^{\alpha}
$$

then $f(x)$ is a Hölder function of exponent $\alpha$ for $x, y \in F$.

Definition 4 (see $[16,17]$ ). If $f(x)$ satisfies the following inequality

$$
\left|f(x)-f\left(x_{0}\right)\right| \leqq o\left(\left(x-x_{0}\right)^{\alpha}\right),
$$

then $f(x)$ is continuous of order $\alpha(0<\alpha \leqq 1)$ or, briefly, $\alpha$-continuous.

Definition 5 (see $[14,16-18]$ ). Suppose that $f(x)$ is local fractional continuous on the interval $(a, b)$; then the local fractional derivative of $f(x)$ of order $\alpha$ at $x=x_{0}$ is given by

$$
\begin{aligned}
f^{(\alpha)}\left(x_{0}\right) & =\left.\frac{d^{\alpha} f(x)}{d x^{\alpha}}\right|_{x=x_{0}} \\
& =\lim _{x \rightarrow x_{0}} \frac{\Gamma(1+\alpha) \Delta\left(f(x)-f\left(x_{0}\right)\right)}{\left(x-x_{0}\right)^{\alpha}},
\end{aligned}
$$

provided this limit exists. [14]):

From Definition 5, we have the following conclusion (see

$$
f^{(\alpha)}(x)=D_{x}^{(\alpha)} f(x),
$$

which is denoted by (see [14])

$$
f(x) \in D_{x}^{(\alpha)}(a, b)
$$

where $D_{x}^{(\alpha)}(a, b)$ denotes the space of local fractional derivable functions on $(a, b)$. 
Definition 6 (see $[14,16-18])$. Suppose that $f(x)$ is local fractional continuous on the interval $(a, b)$; then the local fractional integral of the function $f(x)$ in the interval $[a, b]$ is defined by

$$
\begin{aligned}
{ }_{a} I_{b}^{(\alpha)} f(x) & =\frac{1}{\Gamma(1+\alpha)} \int_{a}^{b} f(t)(d t)^{\alpha} \\
& =\frac{1}{\Gamma(1+\alpha)} \lim _{\Delta t \rightarrow 0} \sum_{j=0}^{N-1} f\left(t_{j}\right)\left(\Delta t_{j}\right)^{\alpha},
\end{aligned}
$$

where $\Delta t_{j}=t_{j+1}-t_{j}, \Delta t=\max \left\{\Delta t_{1}, \Delta t_{2}, \ldots, \Delta t_{j}, \ldots\right\}$, and $\left[t_{j}, t_{j+1}\right]\left(j=1,2, \ldots, N-1 ; t_{0}=a ; t_{N}=b\right)$ are a partition of the interval $[a, b]$.

Let $I_{x}^{(\alpha)}(a, b)$ denote the space of local fractional integrable functions on $(a, b)$; from Definition 6 , we can obtain the following result (see, for details, [14]):

$$
f(x) \in{ }_{a} I_{x}^{(\alpha)}(a, b),
$$

if there exists (see [14])

$$
{ }_{a} I_{x}^{(\alpha)} f(x) .
$$

Remark 7 (see [14]). If we suppose that $f(x) \in D_{x}^{(\alpha)}(a, b)$ or $C_{\alpha}(a, b)$, then we have

$$
f(x) \in{ }_{\alpha} I_{x}^{(\alpha)}(a, b) .
$$

\section{Main Results}

In this section, we state and prove our main results.

Theorem 8. Assume that $\alpha_{k j} \in \mathbb{R}(j=1,2, \ldots, m ; k=$ $1,2, \ldots, s)$,

$$
\sum_{k}^{s} \frac{1}{p_{k}}=1, \quad \sum_{k=1}^{s} \alpha_{k j}=0 .
$$

If $f_{j}(x)>0$ and $f_{j} \in C_{\alpha}(a, b)(j=1,2, \ldots, m)$, then each of the following assertions holds true.

(1) For $p_{k}>0(k=1,2, \ldots, s)$, one has

$$
\begin{aligned}
& \frac{1}{\Gamma(1+\alpha)} \int_{a}^{b} \prod_{j=1}^{m} f_{j}(x)(d x)^{\alpha} \\
& \leqq \prod_{k=1}^{s}\left(\frac{1}{\Gamma(1+\alpha)} \int_{a}^{b} \prod_{j=1}^{m} f_{j}^{1+p_{k} \alpha_{k j}}(x)(d x)^{\alpha}\right)^{1 / p_{k}} .
\end{aligned}
$$

(2) For $0<p_{s}<1$ and $p_{k}<0 \quad(k=1,2, \ldots, s-1)$, one has

$$
\begin{aligned}
& \frac{1}{\Gamma(1+\alpha)} \int_{a}^{b} \prod_{j=1}^{m} f_{j}(x)(d x)^{\alpha} \\
& \geqq \prod_{k=1}^{s}\left(\frac{1}{\Gamma(1+\alpha)} \int_{a}^{b} \prod_{j=1}^{m} f_{j}^{1+p_{k} \alpha_{k j}}(x)(d x)^{\alpha}\right)^{1 / p_{k}} .
\end{aligned}
$$

Proof. (1) Let

$$
g_{k}(x)=\left(\prod_{j=1}^{m} f_{j}^{1+p_{k} \alpha_{k j}}(x)\right)^{1 / p_{k}}
$$

Applying the assumptions $\sum_{k}^{s}\left(1 / p_{k}\right)=1$ and $\sum_{k=1}^{s} \alpha_{k j}=0$, a direct computation shows that

$$
\begin{aligned}
\prod_{k=1}^{s} g_{k}(x)= & g_{1} g_{2} \cdots g_{s} \\
= & \left(\prod_{j=1}^{m} f_{j}^{1+a_{1} \alpha_{1 j}}(x)\right)^{1 / a_{1}}\left(\prod_{j=1}^{m} f_{j}^{1+a_{2} \alpha_{2 j}}(x)\right)^{1 / a_{2}} \\
& \ldots\left(\prod_{j=1}^{m} f_{j}^{1+a_{s} \alpha_{s j}}(x)\right)^{1 / a_{s}} \\
= & \prod_{j=1}^{m} f_{j}^{1 / a_{1}+\alpha_{1 j}}(x) \prod_{j=1}^{m} f_{j}^{1 / a_{2}+\alpha_{2 j}}(x) \cdots \prod_{j=1}^{m} f_{j}^{1 / a_{s}+\alpha_{s j}}(x) \\
= & \prod_{j=1}^{m} f_{j}^{1 / a_{1}+1 / a_{2}+\cdots 1 / a_{s}+\alpha_{1 j}+\alpha_{2 j}+\cdots+\alpha_{s j}}(x) \\
= & \prod_{j=1}^{m} f_{j}(x) ;
\end{aligned}
$$

that is,

$$
\prod_{k=1}^{s} g_{k}(x)=\prod_{j=1}^{m} f_{j}(x) .
$$

It is easy to see that

$$
\frac{1}{\Gamma(1+\alpha)} \int_{a}^{b} \prod_{j=1}^{m} f_{j}(x)(d x)^{\alpha}=\frac{1}{\Gamma(1+\alpha)} \int_{a}^{b} \prod_{k=1}^{s} g_{k}(x)(d x)^{\alpha} .
$$

It follows from the Hölder inequality (6) that

$$
\begin{aligned}
& \frac{1}{\Gamma(1+\alpha)} \int_{a}^{b} \prod_{k=1}^{s} g_{k}(x)(d x)^{\alpha} \\
& \leqq \prod_{k=1}^{s}\left(\frac{1}{\Gamma(1+\alpha)} \int_{a}^{b} g_{k}^{p_{k}}(x)(d x)^{\alpha}\right)^{1 / p_{k}} .
\end{aligned}
$$

Substitution of $g_{k}(x)$ into (30) leads us immediately to inequality (24). This proves inequality (24).

(2) The proof of inequality (25) is similar to the proof of inequality (24). Indeed, by using (26), (29), and (7), we have

$$
\begin{aligned}
& \frac{1}{\Gamma(1+\alpha)} \int_{a}^{b} \prod_{k=1}^{s} g_{k}(x)(d x)^{\alpha} \\
& \geqq \prod_{k=1}^{s}\left(\frac{1}{\Gamma(1+\alpha)} \int_{a}^{b} g_{k}^{p_{k}}(x)(d x)^{\alpha}\right)^{1 / p_{k}} .
\end{aligned}
$$


Substitution of $g_{k}(x)$ into (31) leads to inequality (25) immediately.

Remark 9. Upon setting $s=m, \alpha_{k j}=-1 / p_{k}$, for $j \neq k$, and $\alpha_{k k}=1-1 / p_{k}$, inequalities (24) and (25) are reduced to inequalities (6) and (7), respectively.

As we remarked earlier, many existing inequalities related to the local fractional integral Hölder's inequality are special cases of inequalities (24) and (25). For example, we have the following corollary.

Corollary 10. Under the assumptions of Theorem 8 with $s=$ $m, \alpha_{k j}=-t / p_{k}$, for $j \neq k$, and $\alpha_{k k}=t\left(1-1 / p_{k}\right)(t \in \mathbb{R})$, each of the following assertions holds true.

(1) For $p_{k}>0(k=1,2, \ldots, s)$, one has

$$
\begin{aligned}
& \frac{1}{\Gamma(1+\alpha)} \int_{a}^{b} \prod_{j=1}^{m} f_{j}(x)(d x)^{\alpha} \\
& \leqq \prod_{k=1}^{m}\left(\frac{1}{\Gamma(1+\alpha)} \int_{a}^{b}\left(\prod_{j=1}^{m} f_{j}(x)\right)^{1-t}\left(f_{k}^{p_{k}}\right)^{t}(x)(d x)^{\alpha}\right)^{1 / p_{k}} .
\end{aligned}
$$
has

(2) For $0<p_{m}<1$ and $p_{k}<0(k=1,2, \ldots, m-1)$, one

$$
\begin{aligned}
& \frac{1}{\Gamma(1+\alpha)} \int_{a}^{b} \prod_{j=1}^{m} f_{j}(x)(d x)^{\alpha} \\
& \geqq \prod_{k=1}^{m}\left(\frac{1}{\Gamma(1+\alpha)} \int_{a}^{b}\left(\prod_{j=1}^{m} f_{j}(x)\right)^{1-t}\left(f_{k}^{p_{k}}\right)^{t}(x)(d x)^{\alpha}\right)^{1 / p_{k}} .
\end{aligned}
$$

Theorem 11. Assume that $r \in \mathbb{R}, \alpha_{k j} \in \mathbb{R}(j=$ $1,2, \ldots, m ; k=1,2, \ldots, s)$,

$$
\sum_{k}^{s} \frac{1}{p_{k}}=r, \quad \sum_{k=1}^{s} \alpha_{k j}=0 .
$$

If $f_{j}(x)>0$ and $f_{j} \in C_{\alpha}(a, b)(j=1,2, \ldots, m)$, then each of the following assertions holds true.

(1) For $r p_{k}>0(k=1,2, \ldots, s)$, one has

$$
\begin{aligned}
& \frac{1}{\Gamma(1+\alpha)} \int_{a}^{b} \prod_{j=1}^{m} f_{j}(x)(d x)^{\alpha} \\
& \leqq \prod_{k=1}^{s}\left(\frac{1}{\Gamma(1+\alpha)} \int_{a}^{b} \prod_{j=1}^{m} f_{j}^{1+r p_{k} \alpha_{k j}}(x)(d x)^{\alpha}\right)^{1 / r p_{k}} .
\end{aligned}
$$

(2) For $0<r p_{s}<1$ and $r p_{k}<0(k=1,2, \ldots, s-1)$, one has

$$
\begin{aligned}
& \frac{1}{\Gamma(1+\alpha)} \int_{a}^{b} \prod_{j=1}^{m} f_{j}(x)(d x)^{\alpha} \\
& \geqq \prod_{k=1}^{s}\left(\frac{1}{\Gamma(1+\alpha)} \int_{a}^{b} \prod_{j=1}^{m} f_{j}^{1+r p_{k} \alpha_{k j}}(x)(d x)^{\alpha}\right)^{1 / r p_{k}} .
\end{aligned}
$$

Proof. (1) Since $r p_{k}>0$ and $\sum_{k}^{s}\left(1 / p_{k}\right)=r$, we get $\sum_{k}^{s}\left(1 / r p_{k}\right)=1$. Then, by applying (24), we immediately obtain inequality (35).

(2) Since $0<r p_{s}<1, r p_{k}<0$, and $\sum_{k}^{s}\left(1 / p_{k}\right)=r$, we have $\sum_{k}^{s}\left(1 / r p_{k}\right)=1$. Thus, by applying (25), we immediately have inequality (36). This completes the proof of Theorem 11.

From Theorem 11, we obtain Corollary 12, which is a generalization of Theorem 11 .

Corollary 12. Under the assumptions of Theorem 11, let $s=$ $2, p_{1}=p, p_{2}=q$, and $\alpha_{1 j}=-\alpha_{2 j}=\alpha_{j}$. Then each of the following assertions holds true.

(1) For $r p>0$, one has

$$
\begin{aligned}
& \frac{1}{\Gamma(1+\alpha)} \int_{a}^{b} \prod_{j=1}^{m} f_{j}(x)(d x)^{\alpha} \\
& \leqq \\
& \left(\frac{1}{\Gamma(1+\alpha)} \int_{a}^{b} \prod_{j=1}^{m} f_{j}^{1+r p \alpha_{j}}(x)(d x)^{\alpha}\right)^{1 / r p} \\
& \quad\left(\frac{1}{\Gamma(1+\alpha)} \int_{a}^{b} \prod_{j=1}^{m} f_{j}^{1-r q \alpha_{j}}(x)(d x)^{\alpha}\right)^{1 / r q} .
\end{aligned}
$$

(2) For $0<r p<1$, one has

$$
\begin{aligned}
& \frac{1}{\Gamma(1+\alpha)} \int_{a}^{b} \prod_{j=1}^{m} f_{j}(x)(d x)^{\alpha} \\
& \geqq\left(\frac{1}{\Gamma(1+\alpha)} \int_{a}^{b} \prod_{j=1}^{m} f_{j}^{1+r p \alpha_{j}}(x)(d x)^{\alpha}\right)^{1 / r p} \\
& \quad\left(\frac{1}{\Gamma(1+\alpha)} \int_{a}^{b} \prod_{j=1}^{m} f_{j}^{1-r q \alpha_{j}}(x)(d x)^{\alpha}\right)^{1 / r q} .
\end{aligned}
$$

Next we present a refinement of each of inequalities (35) and (36).

Theorem 13. Under the assumptions of Theorem 11, each of the following assertions holds true.

(1) For $r p_{k}>0(k=1,2, \ldots, s)$, one has

$$
\begin{aligned}
& \frac{1}{\Gamma(1+\alpha)} \int_{a}^{b} \prod_{j=1}^{m} f_{j}(x)(d x)^{\alpha} \\
& \leqq \varphi(c) \leqq \prod_{k=1}^{s}\left(\frac{1}{\Gamma(1+\alpha)} \int_{a}^{b} \prod_{j=1}^{m} f_{j}^{1+r p_{k} \alpha_{k j}}(x)(d x)^{\alpha}\right)^{1 / r p_{k}}
\end{aligned}
$$

where

$$
\begin{aligned}
\varphi(c) \equiv & \frac{1}{\Gamma(1+\alpha)} \int_{a}^{c} \prod_{j=1}^{m} f_{j}(x)(d x)^{\alpha} \\
& +\prod_{k=1}^{s}\left(\frac{1}{\Gamma(1+\alpha)} \int_{c}^{b} \prod_{j=1}^{m} f_{j}^{1+r p_{k} \alpha_{k j}}(x)(d x)^{\alpha}\right)^{1 / r p_{k}}
\end{aligned}
$$

is a nonincreasing function with $a \leqq c \leqq b$. 

has

(2) For $0<r p_{s}<1$ and $r p_{k}<0(k=1,2, \ldots, s-1)$, one

$$
\begin{aligned}
& \frac{1}{\Gamma(1+\alpha)} \int_{a}^{b} \prod_{j=1}^{m} f_{j}(x)(d x)^{\alpha} \\
& \geqq \phi(c) \geqq \prod_{k=1}^{s}\left(\frac{1}{\Gamma(1+\alpha)} \int_{a}^{b} \prod_{j=1}^{m} f_{j}^{1+r p_{k} \alpha_{k j}}(x)(d x)^{\alpha}\right)^{1 / r p_{k}},
\end{aligned}
$$

where

$$
\begin{aligned}
\phi(c) \equiv & \frac{1}{\Gamma(1+\alpha)} \int_{a}^{c} \prod_{j=1}^{m} f_{j}(x)(d x)^{\alpha} \\
& +\prod_{k=1}^{s}\left(\frac{1}{\Gamma(1+\alpha)} \int_{c}^{b} \prod_{j=1}^{m} f_{j}^{1+r p_{k} \alpha_{k j}}(x)(d x)^{\alpha}\right)^{1 / r p_{k}}
\end{aligned}
$$

is a nondecreasing function with $a \leqq c \leqq b$.

Proof. (1) Let

$$
g_{k}(x)=\left(\prod_{j=1}^{m} f_{j}^{1+r p_{k} \alpha_{k j}}(x)\right)^{1 / r p_{k}}
$$

By rearrangement, it follows from the assumptions of Theorem 11 that

$$
\prod_{j=1}^{m} f_{j}(x)=\prod_{k=1}^{s} g_{k}(x) .
$$

Then, by Hölder's inequality (6), we obtain

$$
\begin{aligned}
& \frac{1}{\Gamma(1+\alpha)} \int_{a}^{b} \prod_{j=1}^{m} f_{j}(x)(d x)^{\alpha} \\
= & \frac{1}{\Gamma(1+\alpha)} \int_{a}^{b} \prod_{k=1}^{s} g_{k}(x)(d x)^{\alpha} \\
= & \frac{1}{\Gamma(1+\alpha)} \int_{a}^{c} \prod_{k=1}^{s} g_{k}(x)(d x)^{\alpha}+\frac{1}{\Gamma(1+\alpha)} \int_{c}^{b} \prod_{k=1}^{s} g_{k}(x)(d x)^{\alpha} \\
\leqq & \frac{1}{\Gamma(1+\alpha)} \int_{a}^{c} \prod_{k=1}^{s} g_{k}(x)(d x)^{\alpha} \\
& +\prod_{k=1}^{s}\left(\frac{1}{\Gamma(1+\alpha)} \int_{c}^{b} g_{k}^{r p_{k}}(x)(d x)^{\alpha}\right)^{1 / r p_{k}} \\
\leqq & \prod_{k=1}^{s}\left(\frac{1}{\Gamma(1+\alpha)} \int_{a}^{c} g_{k}^{r p_{k}}(x)(d x)^{\alpha}\right. \\
& \left.+\frac{1}{\Gamma(1+\alpha)} \int_{c}^{b} g_{k}^{r p_{k}}(x)(d x)^{\alpha}\right)^{1 / r p_{k}}
\end{aligned}
$$

$$
\begin{aligned}
& =\prod_{k=1}^{s}\left(\frac{1}{\Gamma(1+\alpha)} \int_{a}^{b} g_{k}^{r p_{k}}(x)(d x)^{\alpha}\right)^{1 / r p_{k}} \\
& =\prod_{k=1}^{s}\left(\frac{1}{\Gamma(1+\alpha)} \int_{a}^{b} \prod_{j=1}^{m} f_{j}^{1+r p_{k} \alpha_{k j}}(x)(d x)^{\alpha}\right)^{1 / r p_{k}} .
\end{aligned}
$$

Hence, the desired result is obtained.

(2) The proof of inequality (41) is similar to the proof of inequality (39), so we omit the details involved.

\section{Concluding Remarks and Observations}

Integral inequalities play a major role in the development of local fractional calculus. In this work, we considered some new generalizations and refinements of the local fractional integral Hölder's inequality and some related results on fractal space. Hölder's inequality was obtained by Yang [14] using local fractional integral. Moreover, the reverse local fractional integral Hölder's inequality was established by Chen [15]. In our present investigation, we have offered further generalizations and refinements of these inequalities by using the local fractional integral which was introduced and investigated by Yang $[14,16,17]$. Special cases of the various results derived in this paper are shown to be related to a number of known results.

For the relevant details about the mathematical, physical, and engineering applications and interpretations of the operators of fractional calculus and local fractional calculus in dealing with the intermediate processes and the intermediate phenomena, the interested reader may be referred to the monographs by Yang [17] and Kilbas et al. [43] (and indeed also to some of the other recent investigations which are cited in this paper).

\section{Conflict of Interests}

The authors declare that they have no conflict of interests.

\section{Authors' Contribution}

This paper is the result of joint work of all authors who contributed equally to the final version of this paper. All authors read and approved the final paper.

\section{Acknowledgments}

This work was supported by the Key Project of Guangxi Social Sciences (no. gxsk201424), the Scientific Research Program Funded by Shaanxi Provincial Education Department (no. 2013JK1139), the China Postdoctoral Science Foundation (no. 2013M542370), the Specialized Research Fund for the Doctoral Program of Higher Education of China (no. 20136118120010), NNSFC (no. 11326161), the Key Project of Science and Technology Research of the Henan Education Department (no. 14A110011), the Education Science fund of the Education Department of Guangxi (no. 2013JGB410), 
NSFC (no. 61362021), the Natural Science Foundation of Guangxi Province (no. 2013GXNSFDA019030), and the Scientific Research Project of Guangxi Education Department (no. YB2014560 and no. LX2014627).

\section{References}

[1] E. F. Beckenbach and R. Bellman, Inequalities, Springer, New York, NY, USA, 1961.

[2] W.-S. Cheung, "Generalizations of Hölder's inequality," International Journal of Mathematics and Mathematical Sciences, vol. 26, no. 1, pp. 7-10, 2001.

[3] D. S. Mitrinović and P. M. Vasić, Analytic Inequalities, Springer, Berlin, Germany, 1970.

[4] G. H. Hardy, J. E. Littlewood, and G. Pólya, Inequalities, Cambridge University Press, London, UK, 2nd edition, 1952.

[5] S. Abramovich, B. Mond, and J. E. Pecaric, "Sharpening Hölder/s Inequality," Journal of Mathematical Analysis and Applications, vol. 196, no. 3, pp. 1131-1134, 1995.

[6] X. Yang, "Refinement of Hölder inequality and application to Ostrowski inequality," Applied Mathematics and Computation, vol. 138, no. 2-3, pp. 455-461, 2003.

[7] X. Yang, "Hölder's inequality," Applied Mathematics Letters, vol. 16, no. 6, pp. 897-903, 2003.

[8] S. H. Wu, "Generalization of a sharp Hölder's inequality and its application," Journal of Mathematical Analysis and Applications, vol. 332, no. 1, pp. 741-750, 2007.

[9] E. G. Kwon and E. K. Bae, "On a continuous form of Hölder inequality," Journal of Mathematical Analysis and Applications, vol. 343, no. 1, pp. 585-592, 2008.

[10] M. Masjed-Jamei, "A functional generalization of the CauchySchwarz inequality and some subclasses," Applied Mathematics Letters, vol. 22, no. 9, pp. 1335-1339, 2009.

[11] W. Yang, "A functional generalization of diamond- $\alpha$ integral Hölder's inequality on time scales," Applied Mathematics Letters, vol. 23 , no. 10, pp. 1208-1212, 2010.

[12] D. K. Callebaut, "Generalization of the Cauchy-Schwarz inequality," Journal of Mathematical Analysis and Applications, vol. 12, pp. 491-494, 1965.

[13] H. Qiang and Z. Hu, "Generalizations of Hölder's and some related inequalities," Computers \& Mathematics with Applications, vol. 61, no. 2, pp. 392-396, 2011.

[14] X.-J. Yang, Local Fractional Functional Analysis and Its Applications, Asian Academic Publisher, Hong Kong, 2011.

[15] G.-S. Chen, "Generalizations of Hölder's and some related integral inequalities on fractal space," Journal of Function Spaces and Applications, vol. 2013, Article ID 198405, 9 pages, 2013.

[16] X.-J. Yang, "Local fractional integral transforms," Progress in Nonlinear Science, vol. 4, pp. 1-225, 2011.

[17] X.-J. Yang, Advanced Local Fractional Calculus and Its Applications, World Science Publisher, New York, NY, USA, 2012.

[18] X.-Y. Yang, D. Baleanu, and J. A. T. Machado, "Mathematical aspects of the Heisenberg uncertainty principle within local fractional Fourier analysis," Boundary Value Problems, vol. 2013, article 131, 2013.

[19] A. M. Yang, X. J. Yang, and Z. B. Li, "Local fractional series expansion method for solving wave and diffusion equations on Cantor sets," Abstract and Applied Analysis, vol. 2013, Article ID 351057, 5 pages, 2013.
[20] Y.-J. Yang, D. Baleanu, and X.-J. Yang, "Analysis of fractal wave equations by local fractional Fourier series method," Advances in Mathematical Physics, vol. 2013, Article ID 632309, 2013.

[21] X.-J. Ma, H. M. Srivastava, D. Baleanu, and X.-J. Yang, "A new Neumann series method for solving a family of local fractional Fredholm and Volterra integral equations," Mathematical Problems in Engineering, vol. 2013, Article ID 325121, 6 pages, 2013.

[22] Y.-J. Hao, H. M. Srivastava, H. Jafari, and X.-J. Yang, "Helmholtz and diffusion equations associated with local fractional derivative operators involving the Cantorian and Cantor-type cylindrical coordinates," Advances in Mathematical Physics, vol. 2013, Article ID 754248, 5 pages, 2013.

[23] S. Umarov and S. Steinberg, "Variable order differential equations with piecewise constant order-function and diffusion with changing modes," Zeitschrift für Analysis und ihre Anwendungen, vol. 28, no. 4, pp. 431-450, 2009.

[24] X. J. Yang, D. Baleanu, and Y. Khan, "Local fractional variational iteration method for diffusion and wave equations on Cantor sets," Romanian Journal of Physics, vol. 59, no. 1-2, pp. 36-48, 2014.

[25] D. Baleanu, J. A. Tenreiro Machado, C. Cattani, M. C. Baleanu, and X. Yang, "Local fractional variational iteration and decomposition methods for wave equation on Cantor sets within local fractional operators," Abstract and Applied Analysis, vol. 2014, Article ID 535048, 6 pages, 2014.

[26] A. Carpinteri, B. Chiaia, and P. Cornetti, "Static-kinematic duality and the principle of virtual work in the mechanics of fractal media," Computer Methods in Applied Mechanics and Engineering, vol. 191, no. 1-2, pp. 3-19, 2001.

[27] A. Carpinteri and P. Cornetti, "A fractional calculus approach to the description of stress and strain localization in fractal media," Chaos, Solitons \& Fractals, vol. 13, no. 1, pp. 85-94, 2002.

[28] A. Atangana and A. Secer, "A note on fractional order derivatives and table of fractional derivatives of some special functions," Abstract and Applied Analysis, vol. 2013, Article ID 279681, 8 pages, 2013.

[29] K. M. Kolwankar and A. D. Gangal, "Local fractional FokkerPlanck equation," Physical Review Letters, vol. 80, no. 2, pp. 214217, 1998.

[30] G. Wu and K.-T. Wu, "Variational approach for fractional diffusion-wave equations on cantor sets," Chinese Physics Letters, vol. 29, no. 6, Article ID 060505, 2012.

[31] W. Zhong, F. Gao, and X. Shen, "Applications of Yang-Fourier transform to local fractional equations with local fractional derivative and local fractional integral," Advanced Materials Research, vol. 461, pp. 306-310, 2012.

[32] A. H. Cloot and J. P. Botha, "A generalized groundwater flow equation using the concept of non-integer order," Water $S A$, vol. 32, no. 1, pp. 1-7, 2006.

[33] M.-S. Hu, R. P. Agarwal, and X.-J. Yang, "Local fractional Fourier series with application to wave equation in fractal vibrating string," Abstract and Applied Analysis, vol. 2012, Article ID 567401, 15 pages, 2012.

[34] M.-S. Hu, D. Baleanu, and X.-J. Yang, "One-phase problems for discontinuous heat transfer in fractal media," Mathematical Problems in Engineering, vol. 2013, Article ID 358473, 3 pages, 2013.

[35] A. Babakhani and V. Daftardar-Gejji, "On calculus of local fractional derivatives," Journal of Mathematical Analysis and Applications, vol. 270, no. 1, pp. 66-79, 2002. 
[36] Y. Chen, Y. Yan, and K. Zhang, "On the local fractional derivative," Journal of Mathematical Analysis and Applications, vol. 362, no. 1, pp. 17-33, 2010.

[37] T. S. Kim, "Differentiability of fractal curves," Korean Mathematical Society: Communications, vol. 20, no. 4, pp. 827-835, 2005.

[38] A. Parvate and A. D. Gangal, "Fractal differential equations and fractal-time dynamical systems," Pramana Journal of Physics, vol. 64, no. 3, pp. 389-409, 2005.

[39] A. V. Chechkin, R. Gorenflo, and I. M. Sokolov, "Fractional diffusion in inhomogeneous media," Journal of Physics A: Mathematical and General, vol. 38, no. 42, pp. L679-L684, 2005.

[40] X.-J. Yang, H. M. Srivastava, J.-H. He, and D. Baleanu, "Cantortype cylindrical-coordinate method for differential equations with local fractional derivatives," Physics Letters A, vol. 377, no. 28-30, pp. 1696-1700, 2013.

[41] H. M. Srivastava, A. K. Golmankhaneh, D. Baleanu, and X. J. Yang, "Local Fractional Sumudu Transform with Application to IVPs on Cantor SETs," Abstract and Applied Analysis, vol. 2014, Article ID 620529, 7 pages, 2014.

[42] W. Wei, H. M. Srivastava, L. Wang, P.-Y. Shen, and J. Zhang, "A local fractional integral inequality on fractal space analogous to Anderson's inequality," Abstract and Applied Analysis, vol. 2014, Article ID 797561, 7 pages, 2014.

[43] A. A. Kilbas, H. M. Srivastava, and J. J. Trujillo, Theory and Applications of Fractional Differential Equations, vol. 204 of North-Holland Mathematics Studies, Elsevier Science, Amsterdam, The Netherlands, 2006. 


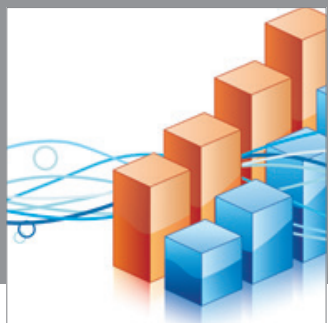

Advances in

Operations Research

mansans

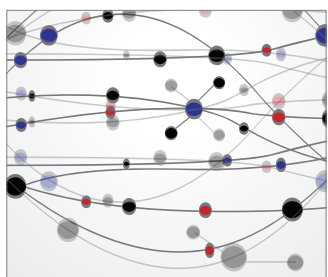

The Scientific World Journal
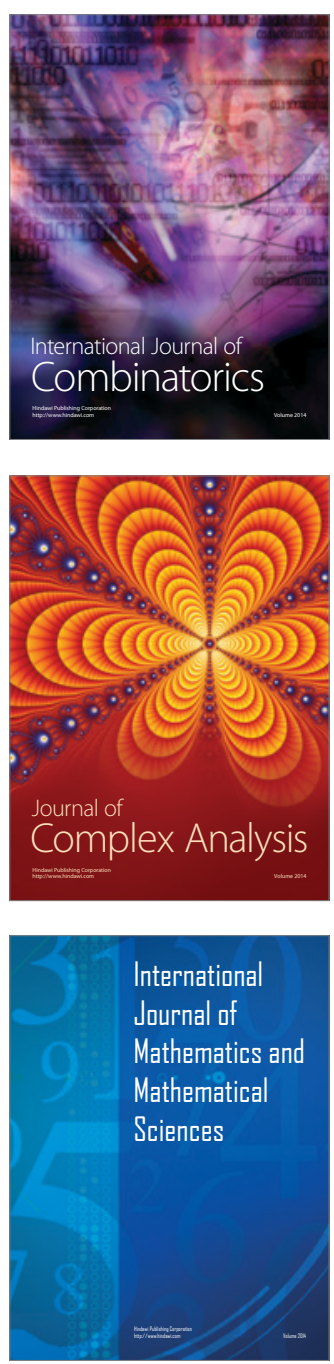
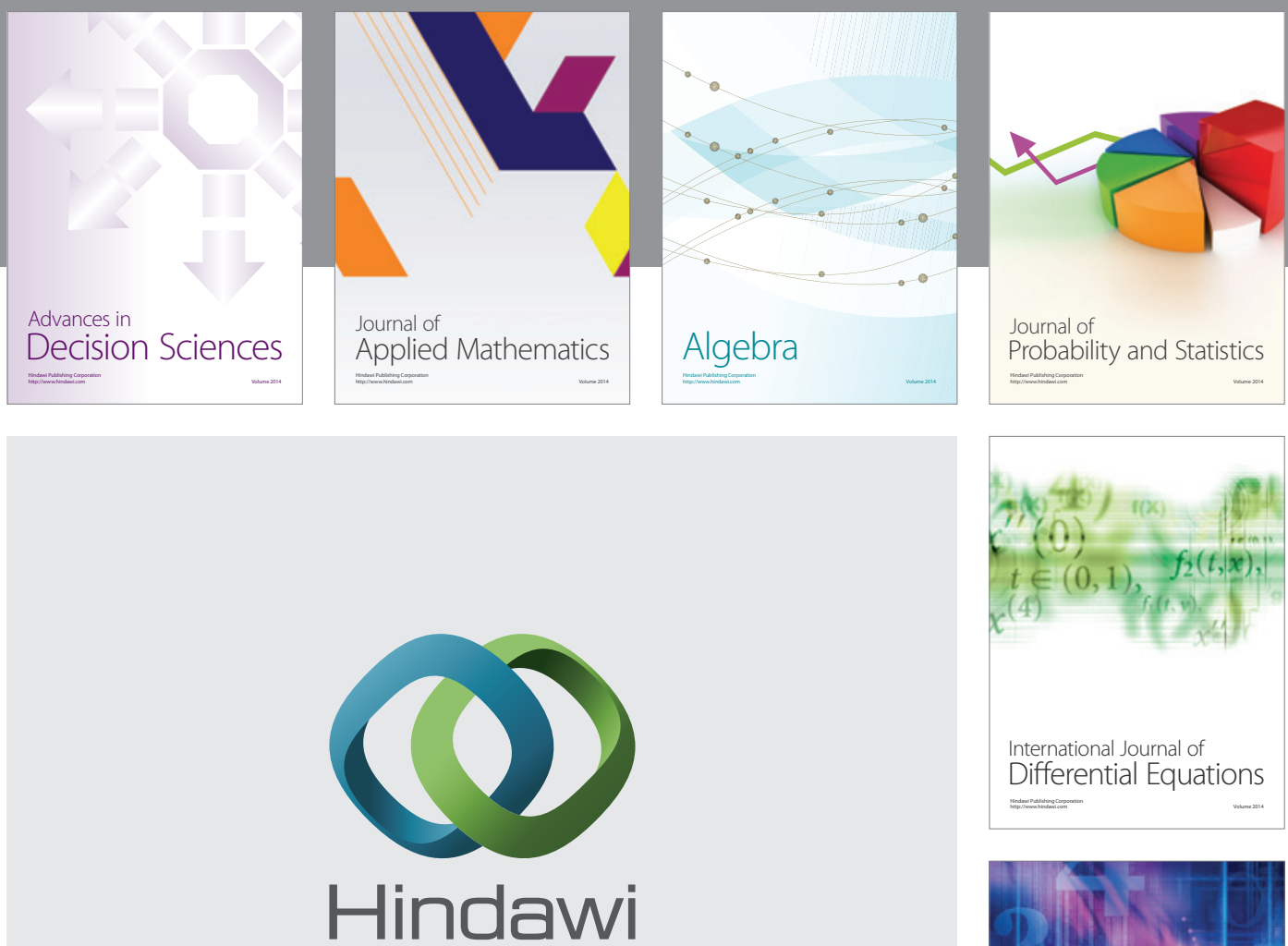

Submit your manuscripts at http://www.hindawi.com
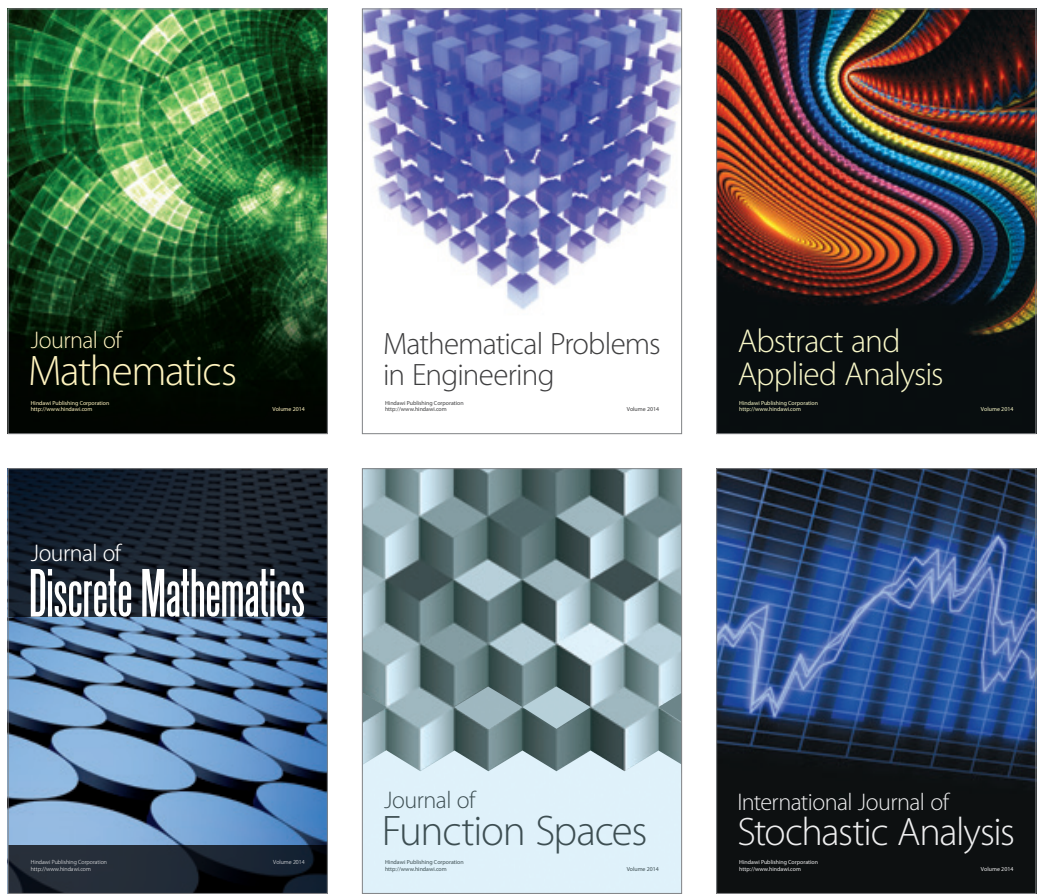

Journal of

Function Spaces

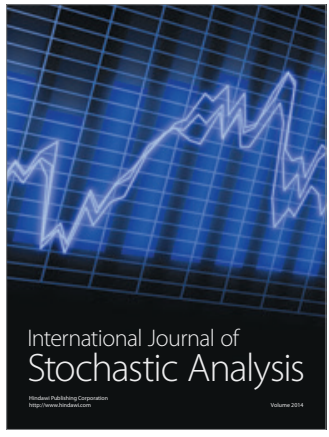

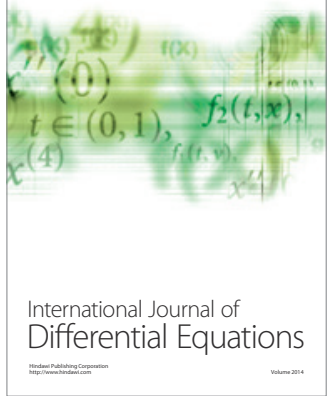
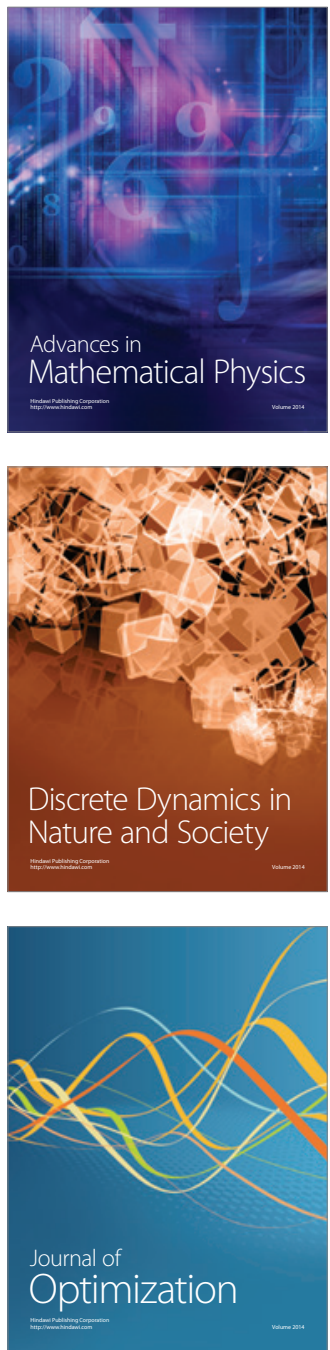